\title{
Seismic Performance Analysis of Large-scale Underground Commercial Space Structure
}

\author{
$\mathrm{Li} \mathrm{Li}^{1,}$, , Deyun Ding ${ }^{2, \mathrm{~b}}$ and Ji Zhao ${ }^{2}$ \\ ${ }^{1}$ Changchun Railway Traffic Group Co., Ltd., Changchun, 130021, China \\ ${ }^{2}$ Beijing Urban Construction Design \& Development Group Co., Limited, Beijing, 100037, China \\ a454257301@qq.com, bdyding2301@163.com
}

\begin{abstract}
Keywords: Large underground space structure, Seismic performance, Time history analysis, Three-dimensional finite element, Storey drift angle.

Abstract. Base on the underground central business district project in the south economic development zone of Changchun, this research studied seismic performance of a large-scale complex underground commercial space structure under the severe earthquake action. A three-dimensional dynamic model was established with the finite element program MIDAS/GTS. The response of the main structure was obtained using time history analysis method, including the storey relative displacement and storey drift angle between adjacent structures. The numerical results show that the structure can satisfy the requirements of the seismic performance under the severe earthquake action. The results could be regarded as a valuable reference in design practice.
\end{abstract}

\section{Introduction}

By December 2015, 116 rail transit lines with a total mileage of $3612 \mathrm{~km}$ have been put into operation in 26 cities of China [1]; more than 5000km new lines in 40 cities have obtained approval construction. As an important part of the urban lifeline engineering, earthquake-resistance of underground engineering causes great attention [2-8]. With the rapid construction of rail transit, more large underground space structure emerged. Since China is an earthquake-prone country, it is quite necessary to conduct seismic analysis of these large underground space structures under earthquake loading, especially after the Wenchuan earthquake in 2008.

Base on the underground central business district project in the south economic development zone of Changchun, this research studied seismic performance of large-scale complex underground commercial space structure in the severe earthquake action. A three-dimensional dynamic model was established with finite element program MIDAS/GTS. The response of the main structure was obtained using time history analysis method, including the displacement difference between adjacent floors and interlayer drift. The numerical results showed that the structure can satisfy the requirements of the seismic performance under severe earthquake action. The conclusion could be reference in design practice.

\section{Project Overview}

The underground central business district project in the south economic development zone of Changchun with a floor area of $48000 \mathrm{~m}^{2}$ includes the station of Metro Line 1, the People's Street passageway and the station of Metro Line 6, as shown in Fig.1.

Underground space project to the three layers of structure: three layer structure for line 6 station platform and underground parking, underground structure for line 1 on the second floor platform layer, under the people's street wear and underground parking, underground structure layer and the underground station for line 1 hall business development, in the plane of line 1 and line 6 is the "T" type change. Overburden depth of the underground structure is only $3 \mathrm{~m}$ as computing control level.

The station of Metro line 1 is an underground three-floor island station with a total length of 342.9 $\mathrm{m}$. The platform is $118 \mathrm{~m}$ long and $13 \mathrm{~m}$ wide. 
The box-shape framework structure with raft foundation uses the stringer and horizontal system as shown in Fig.1.

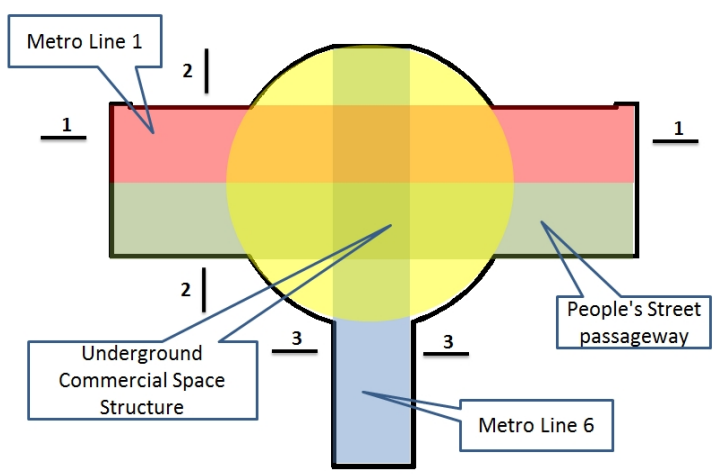

(a) Plan

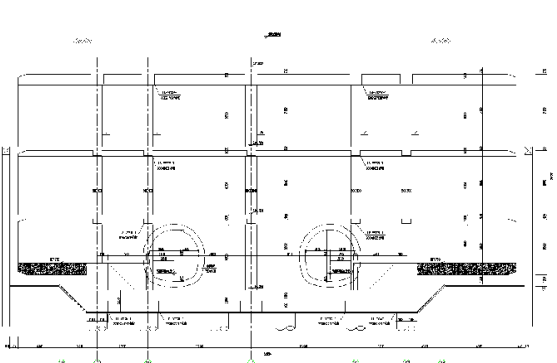

(b) Cross section 3-3

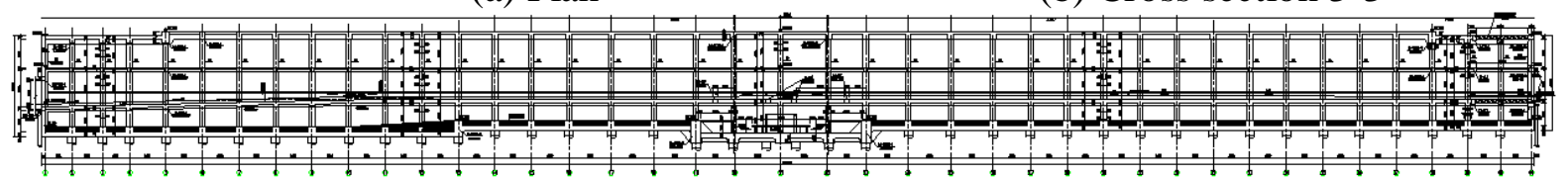

(c) Cross section 1-1

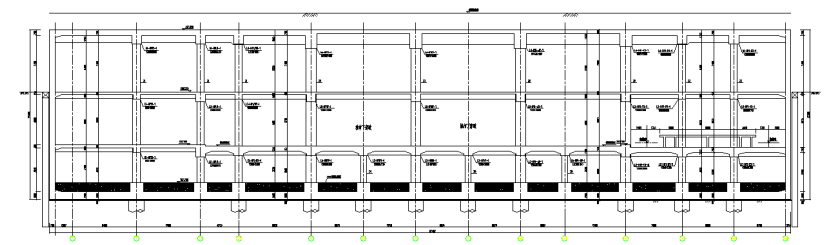

(d) Cross section 2-2

Fig. 1 Plan and cross-section of underground structure

\section{Three-Dimensional Finite Element Model}

Model and Parameters. A three-dimensional finite element dynamic model is established by using the MIDAS/GTS software. To meet the need of analysis, the length (X-direction), width (Y-direction), height(Z-direction) of the model are respectively 630, 550 and $110 \mathrm{~m}$, as shown in Fig.2. The model node number is 108000 and element number is 510000 . There are mainly 4 soil layers in the numerical model on the basis of the geological section map of the station. The soil is modeled by 4-node tetrahedral elements. The soil physical and mechanical parameters are listed in Table 1. The underground structures are represented by the plate elements. Beams, columns and uplift piles were simulated with the beam element. The parameters of structural elements are summarized in Table 2 . The sides and bottom of the model are restricted using the viscoelastic artificial boundary, and the top boundary is free surface.

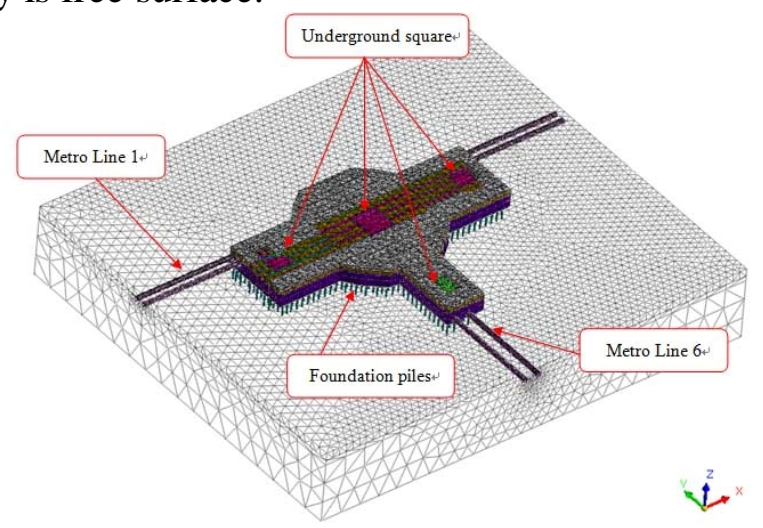

(a) Soil mesh

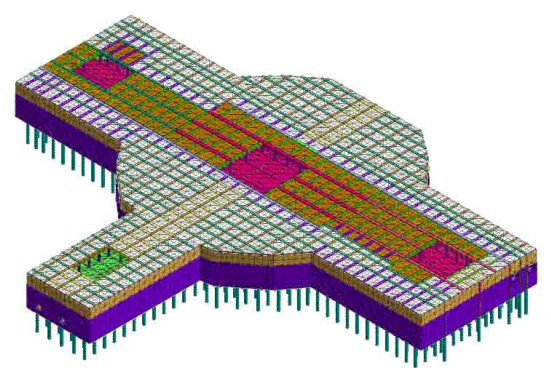

L

(b) Structure mesh

Fig. 2 Three-dimensional finite element model 
Table 1 Physico-mechanical parameters of soils

\begin{tabular}{|c|c|c|c|c|c|c|}
\hline Soil layer & $D[\mathrm{~m}]$ & $E_{\mathrm{d}}[\mathrm{MPa}]$ & $\mu_{\mathrm{d}}[-]$ & $\rho\left[\mathrm{kg} / \mathrm{m}^{3}\right]$ & $V_{\mathrm{s}}[\mathrm{m} / \mathrm{s}]$ & $V_{\mathrm{p}}[\mathrm{m} / \mathrm{s}]$ \\
\hline 1 & 3.9 & 80 & 0.35 & 1950 & 122 & 254 \\
\hline 2 & 6.9 & 60 & 0.38 & 1920 & 105 & 240 \\
\hline 3 & 7.1 & 500 & 0.28 & 2090 & 303 & 548 \\
\hline 4 & 92.1 & 650 & 0.27 & 2110 & 345 & 615 \\
\hline
\end{tabular}

Note: $D$ is thickness; $E_{\mathrm{d}}$ is dynamic elastic modulus; $\mu_{\mathrm{d}}$ is dynamic Poisson's ratio; $\rho$ is density; $v_{\mathrm{s}}$ is shear wave velocity; $v_{\mathrm{p}}$ is compressive wave velocity.

Table 2 Physico-mechanical parameters of structural elements

\begin{tabular}{|c|c|c|c|}
\hline Type & $E[\mathrm{GPa}]$ & $\mu[-]$ & $\rho\left[\mathrm{kg} / \mathrm{m}^{3}\right]$ \\
\hline Main structure & 32.5 & 0.2 & 2500 \\
\hline Column & 34.5 & 0.2 & 2500 \\
\hline Foundation pile & 31.5 & 0.2 & 2500 \\
\hline
\end{tabular}

Note: $E$ is elastic modulus; $\mu$ is Poisson's ratio; $\rho$ is density.

Seismic Loads. In the model, the dynamic equilibrium equation can be expressed by:

$\mathbf{M} \& \mathbf{C} \&+\mathbf{K u}=\mathbf{f}_{t}$

where $\mathbf{M}, \mathbf{C}$ and $\mathbf{K}$ are respectively the mass matrix, damping matrix and stiffness matrix of the system, $\mathbb{\&}$, $\mathbf{U}$ and $\mathbf{u}$ are respectively the acceleration, velocity and displacement of the system, $\mathbf{f}_{t}$ is the seismic load.

The seismic fortification level of the project was set up as 7 degree. Three earthquake acceleration loads with effective peak acceleration $0.2 \mathrm{~g}$ were selected under severe earthquake action. Typical seismic load is shown in Fig.3. The seismic loads were added along the $\mathrm{X}$ axis, $\mathrm{Y}$ axis, and $45^{\circ}$ counterclockwise directions to the $\mathrm{X}$ axis. There are 9 cases considering biaxial loading were simulated in the model.

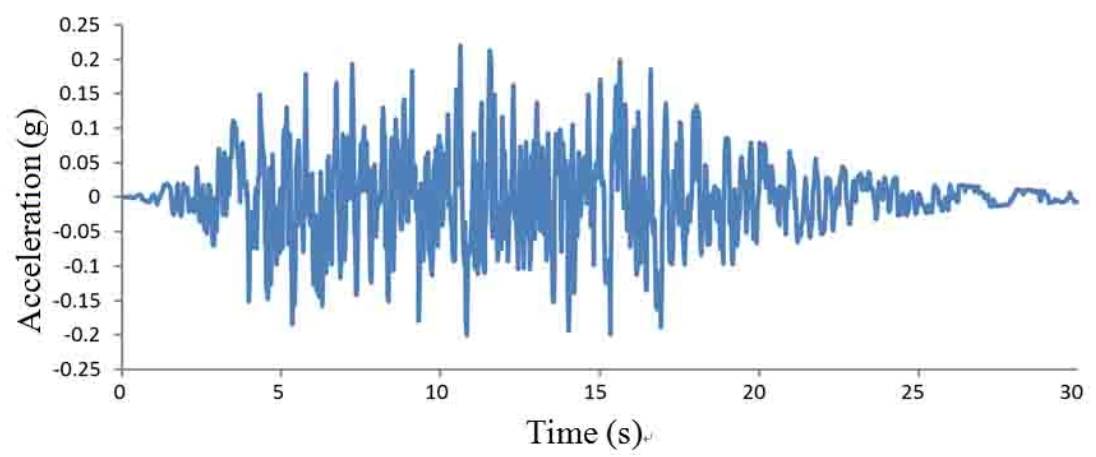

Fig. 3 Typical seismic load

Artificial Boundary. To avoid the reflection of stress wave on the boundary that makes the results distortion, the artificial boundary is used in the three-dimensional dynamic model. The viscoelastic artificial boundary can be used conveniently in the finite element method. The spring elements and damping elements can be placed at the artificial boundary nodes in normal and tangential direction. The values of spring and damping coefficients of three directions are as follows:

Normal: $\quad K_{i x}=\alpha_{x} \frac{G_{i}}{R_{i}} \sum A_{i}, \quad C_{i x}=\rho_{i} c_{i p} \sum A_{i}$

Tangential: $K_{i y}=K_{i z}=\alpha_{y} \frac{G_{i}}{R_{i}} \sum A_{i}=\alpha_{z} \frac{G_{i}}{R_{i}} \sum A_{i}, \quad C_{i y}=C_{i z}=\rho_{i} c_{i s} \sum A_{i}$ 
where $G_{i}$ is medium shear modulus, $\sum A_{i}$ is the area of artificial boundary node $i, R_{i}$ is the distance between load applied point and artificial boundary node $i, \rho_{i}$ is medium density, $c_{i p}$ and $c_{i s}$ respectively are compression wave and shear wave velocity, $\alpha_{\mathrm{x}}, \alpha_{\mathrm{y}}$ and $\alpha_{\mathrm{z}}$ are direction coefficients.

\section{Time-history Result Analysis}

Maximum Horizontal Displacement. The maximum horizontal displacement of underground space structure is $94.08 \mathrm{~mm}$ when the seismic load is in X-axis as shown in Fig. 4(a). The largest deformation is at the side walls of the sinking square in Metro Line 6 station.

The maximum horizontal displacement of underground space structure is $98.48 \mathrm{~mm}$ when the seismic load is in Y-axis as shown in Fig. 4(b). The largest deformation is at roof of first floor in the sinking square.

The maximum horizontal displacement of underground space structure is $99.49 \mathrm{~mm}$ when the seismic load is in Y-axis as shown in Fig. 4(c). The largest deformation is at the top of side walls of the island.

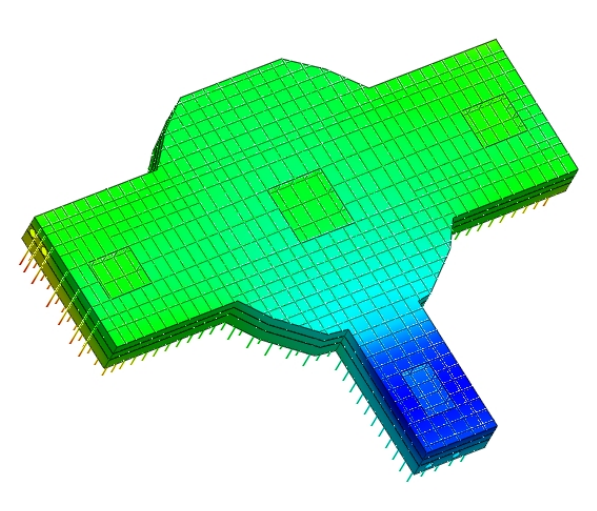

(a) $\mathrm{X}$-axis direction

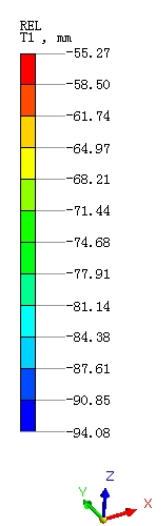

$\stackrel{2}{2}$

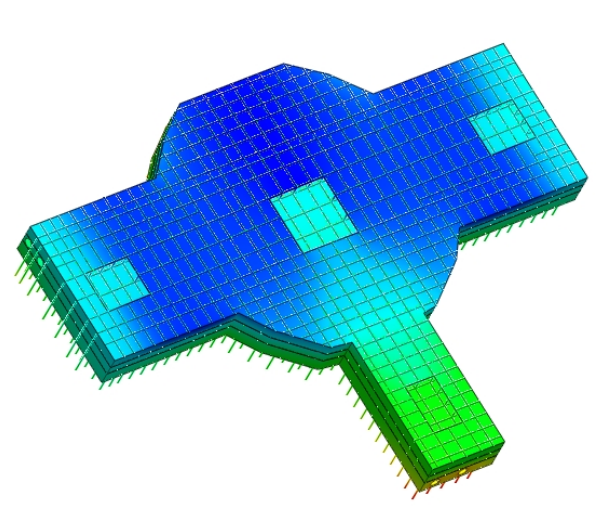

(b) Y-axis direction
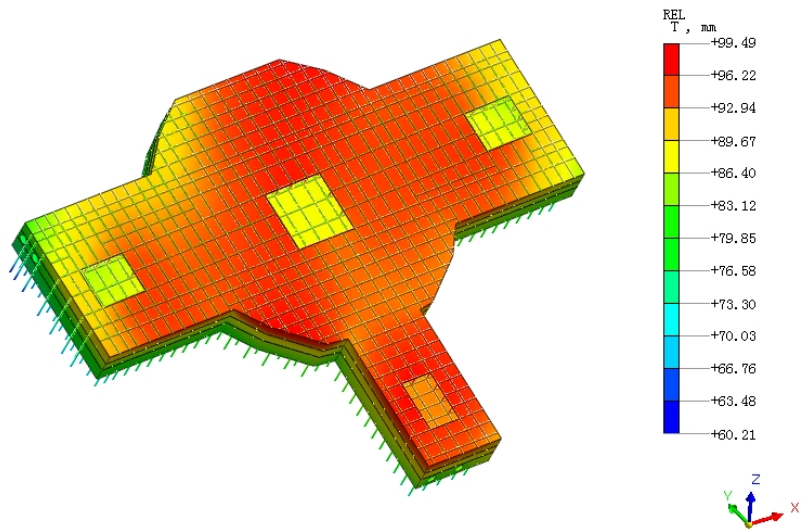

(c) $45^{\circ}$ direction

Fig. 4 Maximum horizontal displacement of the structure under seismic loads

Storey Relative Displacement. In order to analyze the storey relative displacement and storey drift angle between adjacent structures, 3 typical sections of the underground structure were chosen to analyze the data, as shown in Fig.5. Section 7.7 of " Code for seismic design of urban rail transit structures " (GB50909-2014) points out: level II for seismic performance requirements should check structure overall deformation performance; the storey drift angle should be adopted as the index for rectangular cross section structure and the storey drift angle limit of reinforced concrete structures is $1 / 250$ [9]. Then the project in this study was required to check the whole deformation II performance calculation. 


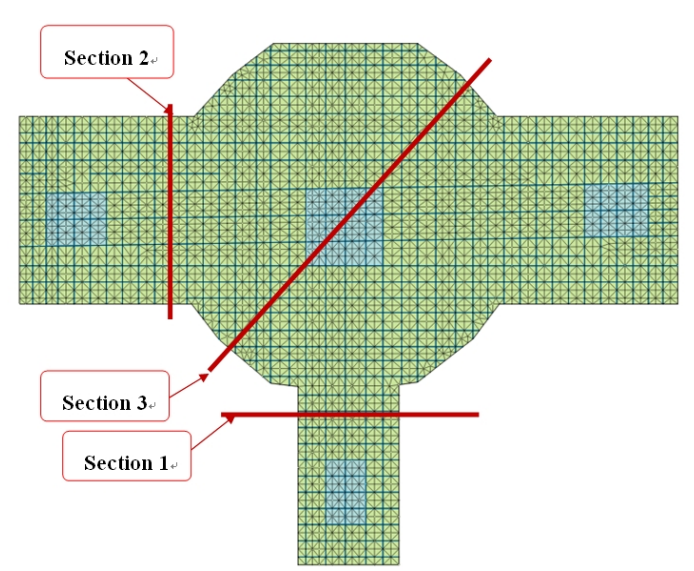

Fig. 5 Typical sections of the underground structure

Fig. 6 shows the storey relative displacement between the roof and bottom for sections 1-3.

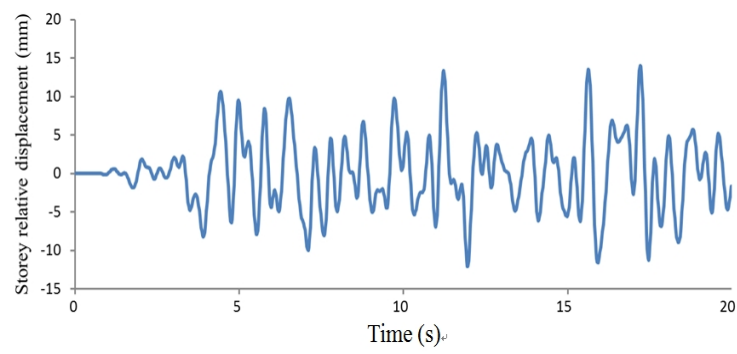

(a) Section 1

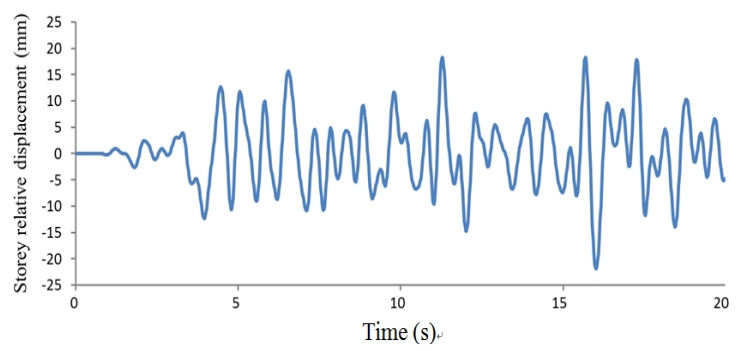

(b) Section 2

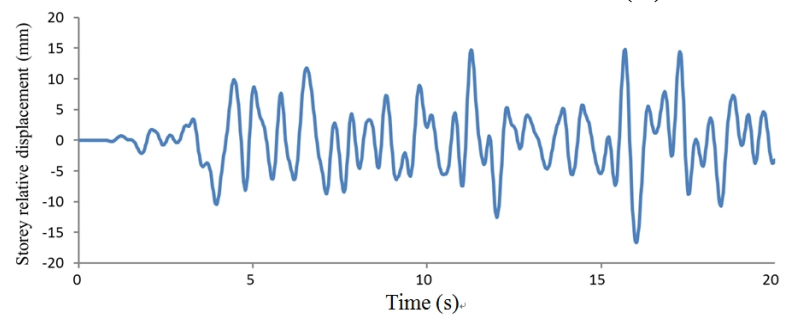

(c) Section 3

Fig. 6 Storey relative displacement between the roof and bottom

Table 3 summarizes the maximums of storey relative displacement and storey drift angle at 3 sections. It is shown that the maximum of storey relative displacement between the roof and bottom is $21.82 \mathrm{~mm}$ and maximum of storey drift angle of the main structure is $1 / 1145$, which is much smaller than the limit 1/250. Results show that the project can meet the requirements of the seismic performance of level II under severe earthquake action.

Table 3 Maximum of storey relative displacement and storey drift angle

\begin{tabular}{|c|c|c|c|c|c|c|}
\hline \multirow{2}{*}{ Load direction } & \multicolumn{3}{|c|}{$\begin{array}{c}\text { Maximum of storey relative } \\
\text { displacement [mm] }\end{array}$} & \multirow{2}{*}{$\begin{array}{c}\text { Maximum } \\
{[\mathrm{mm}]}\end{array}$} & \multicolumn{2}{c|}{$\begin{array}{c}\text { Maximum of storey drift } \\
\text { angle }\end{array}$} \\
\cline { 2 - 4 } & Load 1 & Load 2 & Load 3 & & value & limit \\
\hline X-axis & 14.23 & 14.46 & 14.03 & & & \\
\hline Y-axis & 20.35 & 19.42 & 21.82 & 21.82 & $1 / 1145$ & $1 / 250$ \\
\hline $45^{\circ}$ direction & 16.32 & 16.40 & 16.70 & & & \\
\hline
\end{tabular}

\section{Conclusions}

Base on the underground central business district project in the south economic development zone of Changchun, this research studied seismic performance of large-scale complex underground commercial space structure in the severe earthquake action. The following conclusions can be drawn: 
(1) The maximal displacement of the large underground structure is $99.49 \mathrm{~mm}$. The maximum storey relative displacement can reach $21.82 \mathrm{~mm}$, while the storey drift angle can develop as big as $1 / 1145$.

(2) Under the affection of severe earthquake action, the large underground structure can satisfy the requirements of the seismic performance of level II.

\section{Acknowledgements}

This work is financially supported by the Technology Innovation Project of Beijing Urban Construction Design \& Development Group Co., Limited (Grant No. 2015-18).

\section{References}

[1] Information on http://www.camet.org.cn/

[2] Y.M.A. Hashash, J.J. Hook, B. Schmidt, et al.: Tunneling and Underground Space Technology, Vol. 16 (4) (2001), p. 247.

[3] Shaoping Sun, Yang Han: China Civil Engineering Journal, Vol. 36 (5) (2003), p. 97.

[4] Yu'e Zhang, Baohong Bai, Yaohui Zhang, et al.: Journal of Vibration and Shock, Vol. 22(1) (2003), p. 70.

[5] Jingbo Liu and Bin Li: China Civil Engineering Journal, Vol. 39(6) (2006), p. 106.

[6] Lin Song, Zhaobo Meng, Minzhe Wu, et al.: World Earthquake Engineering, Vol. 26(2) (2010), p.187.

[7] Deyun Ding, Weifeng Wang, Meiqun Huang, et al.: Applied Mechanics and Materials, Vols. 256-259 (2013), p. 2216.

[8] Deyun Ding: Applied Mechanics and Materials, Vols. 501-504 (2014), p. 1524.

[9] Ministry of Housing and Urban-Rural Development of the People's Republic of China: Code for Seismic Design of Urban Rail Transit Structures, GB50909-2014, (2014). 